\title{
Are Code Examples on an Online Q\&A Forum Reliable?
}

\author{
A Study of API Misuse on Stack Overflow
}

\author{
Tianyi Zhang ${ }^{1}$ Ganesha Upadhyaya ${ }^{2}$ Anastasia Reinhardt ${ }^{3 *}$ Hridesh Rajan $^{2}$ Miryung Kim ${ }^{1}$ \\ ${ }^{1}$ University of California, Los Angeles \\ ${ }^{2}$ Iowa State University \\ ${ }^{3}$ George Fox University \\ \{tianyi.zhang, miryung\}@cs.ucla.edu, \{ganeshau, hridesh\}@iastate.edu, areinhardt14@georgefox.edu
}

\begin{abstract}
Programmers often consult an online Q\&A forum such as Stack Overflow to learn new APIs. This paper presents an empirical study on the prevalence and severity of API misuse on Stack Overflow. To reduce manual assessment effort, we design ExAMPLECHECK, an API usage mining framework that extracts patterns from over $380 \mathrm{~K}$ Java repositories on GitHub and subsequently reports potential API usage violations in Stack Overflow posts. We analyze 217,818 Stack Overflow posts using ExAMPLECHECK and find that $31 \%$ may have potential API usage violations that could produce unexpected behavior such as program crashes and resource leaks. Such API misuse is caused by three main reasons-missing control constructs, missing or incorrect order of API calls, and incorrect guard conditions. Even the posts that are accepted as correct answers or upvoted by other programmers are not necessarily more reliable than other posts in terms of API misuse. This study result calls for a new approach to augment Stack Overflow with alternative API usage details that are not typically shown in curated examples.
\end{abstract}

\section{CCS CONCEPTS}

- General and reference $\rightarrow$ Empirical studies; - Software and its engineering $\rightarrow$ Software reliability; Collaboration in software development;

\section{KEYWORDS}

online Q\&A forum, API usage pattern, code example assessment

\section{ACM Reference Format:}

Tianyi Zhang, Ganesha Upadhyaya, Anastasia Reinhardt, Hridesh Rajan, Miryung Kim. 2018. Are Code Examples on an Online Q\&A Forum Reliable?. In Proceedings of ICSE '18: 40th International Conference on Software Engineering, Gothenburg, Sweden, May 27-fune 3, 2018 (ICSE '18), 11 pages. https://doi.org/10.1145/3180155.3180260

*Anastasia Reinhardt contributed to this work as a summer intern at UCLA.

Permission to make digital or hard copies of all or part of this work for personal or classroom use is granted without fee provided that copies are not made or distributed for profit or commercial advantage and that copies bear this notice and the full citation on the first page. Copyrights for components of this work owned by others than the author(s) must be honored. Abstracting with credit is permitted. To copy otherwise, or republish, to post on servers or to redistribute to lists, requires prior specific permission and/or a fee. Request permissions from permissions@acm.org.

ICSE '18, May 27-fune 3, 2018, Gothenburg, Sweden

(c) 2018 Copyright held by the owner/author(s). Publication rights licensed to Association for Computing Machinery.

ACM ISBN 978-1-4503-5638-1/18/05 ..\$15.00

https://doi.org/10.1145/3180155.3180260

\section{INTRODUCTION}

Library APIs are becoming the fundamental building blocks in modern software development. Programmers reuse existing functionalities in well-tested libraries and frameworks by stitching API calls together, rather than building everything from scratch. Online Q\&A forums such as Stack Overflow have a large number of curated code examples $[22,30]$. Though such curated examples can serve as a good starting point, they could potentially impact the quality of production code, when integrated to a target application verbatim. Recently, Fischer et al. find that $29 \%$ of security-related snippets in Stack Overflow are insecure and these snippets could have been reused by over 1 million Android apps on Google play, which raises a big security concern [9]. Previous studies have also investigated the quality of online code examples in terms of compilability [23, 37], unchecked obsolete usage [39], and comprehension issues [29]. However, none of these studies have investigated the reliability of online code examples in terms of API usage correctness. There is also no tool support to help developers easily recognize unreliable code examples in online Q\&A forums.

This paper aims to assess the reliability of code examples on Stack Overflow by contrasting them against desirable API usage patterns mined from GitHub. Our insight is that commonly recurring API usage from a large code corpus may represent a desirable pattern that a programmer can use to assess or enhance code examples on Stack Overflow. The corpus should be large enough to provide sufficient API usage examples and to mine representative API usage patterns. We also believe that quantifying how many snippets are similar (or related but not similar) to a given example can improve developers' confidence about whether to trust the example as is.

Therefore, we design an API usage mining technology, ExAMPLECHECK that scales to over $380 \mathrm{~K}$ GitHub repositories without sacrificing the fidelity and expressiveness of the underlying API usage representation. By leveraging an ultra-large-scale software mining infrastructure [7, 31], EXAMPLECHECK efficiently searches over GitHub and retrieves an average of 55144 code snippets for a given API within 10 minutes. We perform program slicing to remove statements that are not related to the given API, which improves accuracy in the mining process (Section 5). We combine frequent subsequence mining and SMT-based guard condition mining to retain important API usage features, including the temporal ordering of related API calls, enclosing control structures, and guard conditions that protect an API call. In terms of our study scope, we target 100 Java and Android APIs that are frequently discussed on Stack Overflow. We then inspect all patterns learned by ExAMPLECHECK, create a data set of 180 desirable API usage patterns for the 100 APIs, and study the extent of API misuse in Stack Overflow. 


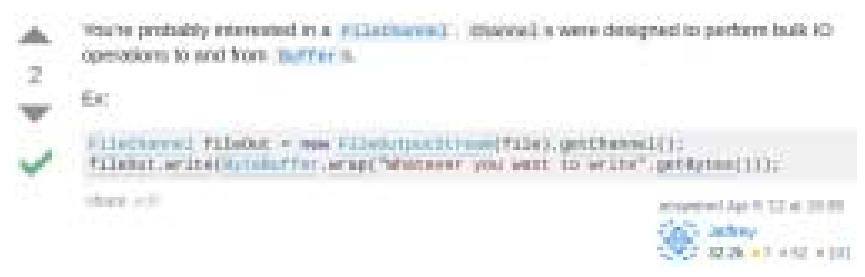

(a) An example that does not close FileChannel properly ${ }^{1}$

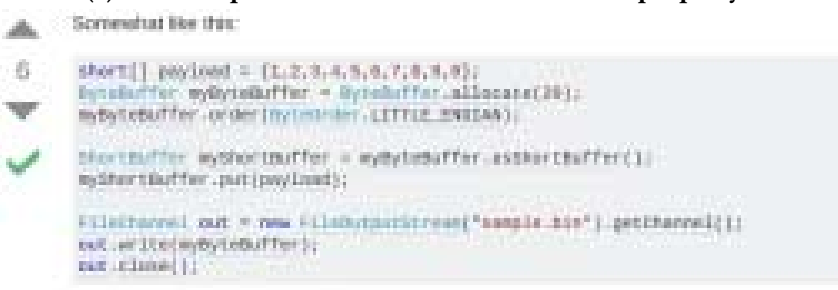

(b) An example that misses exception handling ${ }^{2}$

Figure 1: Two code examples about how to write data to a file using FileChannel on Stack Overflow

Out of 217,818 SO posts relevant to our API data set, $31 \%$ contain potential API misuse that could produce symptoms such as program crashes, resource leaks, and incomplete actions. Such API misuse is caused by three main reasons-missing control constructs, missing or incorrect order of API calls, and incorrect guard conditions. Database, crypto, and networking APIs are often misused, since they often require observing the ordering between multiple calls and complex exception handling logic. Though programmers often put more trust on highly voted posts in Stack Overflow, we do not observe a strong positive nor negative correlation between the number of votes and the reliability of Stack Overflow posts in terms of API usage correctness. This observation suggests that votes alone should not be used as the single indicator of the quality of Stack Overflow posts. Our study provides empirical evidence about the prevalence and severity of API misuse in online Q\&A posts and indicates that Stack Overflow needs another mechanism that helps users to understand the limitation of existing curated examples. We propose a Chrome extension that suggests desirable or alternative API usage for a given Stack Overflow code example, along with supporting concrete examples mined from GitHub.

\section{MOTIVATING EXAMPLES}

Suppose Alice wants to write data to a file using Filechannel. Alice searches on Stack Overflow and finds two code examples, both of which are accepted as correct answers and upvoted by other programmers, as shown in Figure 1. Though such curated examples can serve as a good starting point for API investigation, both examples have API usage violations that may induce unexpected behavior in real applications. If Alice puts too much trust on the given example as is, she may inadvertently follow less ideal API usage.

The first post in Figure 1a does not call FileChannel.close to close the channel. If Alice copies this example to a program that

$\overline{{ }^{1} \text { http://stackoverflow.com/questions/10065852 }}$

${ }^{2}$ http://stackoverflow.com/questions/10506546 does not heavily access new file resources, this example may behave properly, because OS will clean up unmanaged file resources eventually after the program exits. However, if Alice reuses the example in a long-running program with heavy IO, such lingering file resources may cause file handle leaks. Since most operating systems limit the number of opened files, unclosed file streams can eventually run out of file handle resources [28]. Alice may also lose cached data in the file stream, if she uses Filechannel to write a big volume of data but forgots to flush or close the channel.

Even though the second example in Figure $1 \mathrm{~b}$ calls Filechannel.close, it does not handle the potential exceptions thrown by FileChannel.write. Calling write could throw ClosedChannelException, if the channel is already closed. If Alice uses FileChannel in a concurrent program where multiple threads attempt to access the same channel, AsynchronousCloseException will occur if one thread closes the channel, while another thread is still writing data.

As a novice programmer, Alice may not easily recognize the potential limitation of given Stack Overflow examples. In this case, our approach EXAMPLECHECK scans over 380K GitHub repositories and finds 2230 GitHub snippets that also call FileChannel.write. EXAMPLECHECK then learns two common usage patterns from these relevant GitHub snippets. The mostly frequent usage supported by 1829 code snippets on GitHub indicates that a method call to write() must be contained inside a try and catch block. Another frequent usage supported by 1267 GitHub snippets indicates that write must be followed by close. By comparing code snippets in Figures $1 \mathrm{a}$ and $1 \mathrm{~b}$ against these two API usage patterns, Alice may consider adding a missing call to close and an exception handling block during the example integration and adaptation.

\section{API USAGE MINING AND PATTERN SET}

As it is difficult to know desirable or alternative API usage a priori, we design an API usage mining approach, called EXAMPLECHECK that scales to massive code corpora such as GitHub. We then inspect the results manually and construct a data set of desirable API usage to be used for the Stack Overflow study in Section 4.

In terms of API scope, we target 100 popular Java APIs. From the Stack Overflow dump taken in October $2016,{ }^{3}$ we scan and parse all Java code snippets and extract API method calls. We rank the API methods based on frequency and remove trivial ones such as System. out.println. As a result, we select 70 frequently used API methods on Stack Overflow. They are in diverse domains, including Android, Collection, document processing (e.g., String, XML, JSON), graphical user interface (e.g., swing), IO, cryptography, security, Java runtime (e.g. Thread, Process), database, networking, date, and time. The rest 30 APIs come from an API misuse benchmark, MUBENCH [2], after we exclude those patterns without corresponding SO posts and those that cannot be generalized to other projects.

Given an API method of interest, EXAMPLECHECK takes three phases to infer API usage. In Phase 1, given an API method of interest, EXAMPLECHECK searches GitHub snippets that call the given API method, removes irrelevant statements via program slicing, and extracts API call sequences. In Phase 2, ExAmpleCHeck finds common subsequences from individual sequences of API calls. In Phase 3, to retain conditions under which each API can be invoked,

\footnotetext{
${ }^{3}$ https://archive.org/details/stackexchange, accessed on Oct 17, 2016.
} 


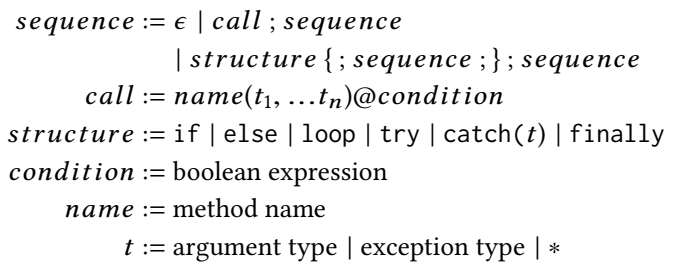

Figure 2: Grammar of Structured API Call Sequences

EXAMPLECHECK mines guard conditions associated with individual API calls. In order to accurately estimate the frequency of unique guard conditions, EXAMPLECHECK uses a SMT solver, Z3 [6], to check the semantic equivalence of guard conditions, instead of considering the syntactic similarity between them only. We manually inspect all inferred patterns to construct the data set of desirable API usage. This data set is used to report potential API misuse in the Stack Overflow posts in our study discussed in Section 4.

\subsection{Structured Call Sequence Extraction and Slicing on GitHub}

Given an API method of interest, ExAMPLECHECK searches individual code snippets invoking the same method in the GitHub corpora. EXAMPLECHECK scans 380,125 Java repositories on GitHub, collected on September 2015. To filter out low-quality GitHub repositories, we only consider repositories with at least 100 revisions and 2 contributors. To scale code search to massive corpora, EXAMPLECHECK leverages a distributed software mining infrastructure [7] to traverse the abstract syntax trees (ASTs) of Java files. EXAMPLECHECK visits every AST method and looks for a method invocation of the API of interest. Figure 3 shows a code snippet retrieved from GitHub for the File.createNewFile API. This snippet creates a property file, if it does not exist by calling createNewFile (line 18).

To extract the essence of API usage, ExAmpLeCHeck models each code snippet as a structured call sequence, which abstracts away certain syntactic details such variable names, but still retains the temporal ordering, control structures, and guard conditions of API calls in a compact manner. Figure 2 defines the grammar of our API usage representation. A structured call sequence consists of relevant control structures and API calls, separated by the delimiter ";". This delimiter is is a separator in our pattern grammar in Figure 2, not a semi-colon for ending each statement in Java. We resolve the argument types of each API call to distinguish method overloading. In certain cases, the argument consists of a complex expression such as write(e.getFormat()), where the partial program analysis may not be able to resolve the corresponding type. In that case, we represent unresolved types with $*$, which can be matched with any other types in the following mining phases. Each API call is associated with a guard condition that protects its usage or true, if it is not guarded by any condition. Catch blocks are also annotated with the corresponding exception types. We normalize a catch block with multiple exception types such as catch (IOException | SQLException) $\{\ldots\}$ to multiple catch blocks with a single exception type such as catch (IOException) $\{\ldots\}$ catch (SQLException) $\{\ldots\}$.

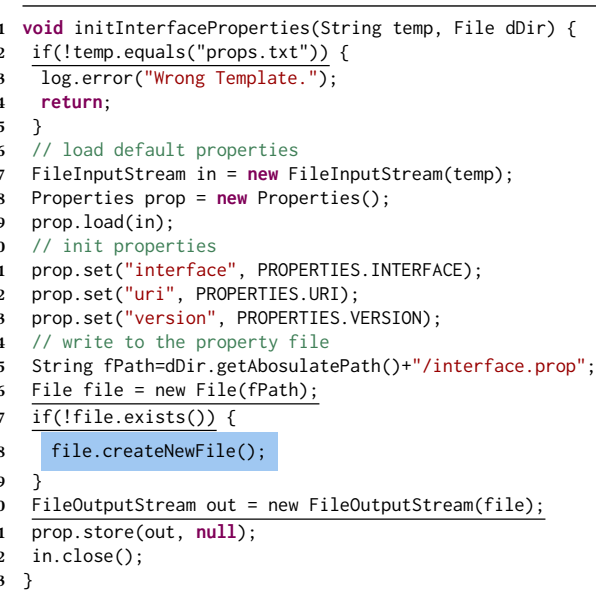

Figure 3: This method is extracted as an example of File.createNewFile from the GitHub copora. Program slicing only retains the underlined statements when $k$ bound is set to 1 , since they have direct control or data dependences on the focal API call to createNewFile at line 18.

EXAMPLECHECK builds the control flow graph of a GitHub snippet and identifies related control structures [1]. A control structure is related to the given API call, if there exists a path between the two and the API call is not post-dominated by the control structure. For instance, the API call to createNewFile (line 18) is control dependent on the if statements at lines 2 and 17 in Figure 3. From each control structure, we lift the contained predicate. This process is a pre-cursor for mining a common guard condition that protects each API method call in Phase 3. We use the conjunction of the lifted predicates in all relevant control structures. If an API call is in the false branch of a control structure, we negate the predicate when constructing the guard. In Figure 3, since createNewFile is in the false branch of the if statement at line 2 and the true branch of the if statement at line 17, its guard condition is temp.equals("props.txt") \&\& !file.exists(). The process of lifting control predicates can be further improved via symbolic execution to account for the effect of program statement before an API call. Project-specific predicates and variable names used in the guard conditions are later generalized in Phase 3 to unify equivalent guards regardless of project-specific details.

EXAMPLECHECK performs intra-procedural program slicing [36] to filter out any statements not related to the API method of interest. For example, Properties API calls in Figure 3 should be removed, since they are irrelevant to createNewFile. During this process, EXAMPLECHECK uses both backward and forward slicing to identify data-dependent statements up to $k$ hops. Setting $k$ to 1 retains only immediately dependent API calls in the call sequence, while setting $k$ to $\infty$ includes all transitively dependent API calls. For instance, the Properties APIs such as load (line 9) and set (lines 1113) are transitively dependent on createNewFile through variables file, out, and prop. Table 1 shows the call sequences extracted from Figure 3 with different $k$ bounds. By removing irrelevant statements, program slicing significantly reduces the mining effort 


\begin{tabular}{|c|c|c|}
\hline Bound & Variables & Structured Call Sequence \\
\hline $\mathrm{k}=1$ & file & new File; if $\{$; createNewFile; $\} ;$ new FileOutputStream \\
\hline $\mathrm{k}=2$ & $\begin{array}{l}\text { file, fPath, } \\
\text { out }\end{array}$ & $\begin{array}{l}\text { getAbsolutePath; new File; if }\{; \text { createNewFile; }\} \\
\text { new FileOutputStream; store }\end{array}$ \\
\hline $\mathrm{k}=3$ & $\begin{array}{l}\text { file, fPath, } \\
\text { out, prop }\end{array}$ & $\begin{array}{l}\text { new Properties; load; set; set; set; getAbsolutePath; new File; } \\
\text { if \{; createNewFile; \}; new FileOutputStream; store }\end{array}$ \\
\hline $\mathrm{k}=\infty$ & $\begin{array}{l}\text { file, fPath, } \\
\text { out, prop, } \\
\text { in, temp }\end{array}$ & $\begin{array}{l}\text { new FileInputStream; new Properties; load; set; set; set; } \\
\text { getAbsolutePath; new File; if \{; createNewFile; \}; } \\
\text { new FileOutputStream; store; close }\end{array}$ \\
\hline No Slicing & $\begin{array}{l}\text { file, fPath, } \\
\text { out, prop, in, } \\
\text { temp, log }\end{array}$ & $\begin{array}{l}\text { if \{; debug; \}; new FileInputStream; new Properties; } \\
\text { getAbsolutePath; load; set; set; set; new File; if \{; } \\
\text { createNewFile; \}; new FileOutputStream; store; close }\end{array}$ \\
\hline
\end{tabular}

Table 1: Structured call sequences sliced using $k$ bounds. Guard conditions and argument types are omitted for presentation purposes.

and also improves the mining precision. Setting $k$ to 1 leads to best performance empirically (discussed in Section 5).

\subsection{Frequent Subsequence Mining}

Given a set of structured call sequences from Phase 1, EXAMPLECHECK finds common subsequences using BIDE [34]. Computing the common subsequence is widely practiced in the literature of API usage mining $[25,26,33,38]$ and has the benefit of filtering out API calls pertinent to only a few outlier examples. In this phase, ExAMPLECHECK focuses on mining the temporal ordering of API calls only. The task of mining a common guard condition is done in Phase 3 instead. BIDE mines frequent closed sequences above a given minimum support threshold $\sigma$. A sequence is a frequent closed sequence, if it occurs frequently above the given threshold and there is no super-sequence with the same support. When matching API signature, EXAMPLECHECK matches $*$ with any other types in the same position in an API call. For example, write(int,*) can be matched with write(int, String) but will not be matched with write(String, int). EXAMPLECHECK ranks a list of sequence patterns based on the number of supporting GitHub examples, which we call support. EXAMPLECHECK filters invalid sequence patterns that do not follow the grammar in Figure 2, as frequent sub-sequence mining can find invalid patterns with unbalanced brackets such as "foo@true; \}; \}".

\subsection{Guard Condition Mining}

Given a common subsequence from Phase 2, ExAMPLECHEck mines the common guard condition of each API call in the sequence. The rationale is that each method call in the common subsequence may have a guard to ensure that the constituent API call does not lead to a failure. Therefore, ExAMPLECHEck collects all guard conditions from each call from Phase 1 and clusters them based on semantic equivalence. The guard conditions extracted from GitHub often contain project-specific predicates and variable names. In Figure 3, the identified guard condition of createNewFile (line 18) is temp.equals("props.txt") \&\& !file.exists(). Its first predicate temp.equals("props.txt") checks whether a string variable temp contains a specific content. Neither the variable temp nor the predicate are related to the usage of createNewFile. Therefore, ExAMPLECHECK first abstracts away such syntactic details before clustering guard conditions. For each guard condition from Phase 1,

\begin{tabular}{|l|l|l|l|}
\hline API Call & Guard & Generalized & Symbolized \\
\hline s.substring(start) & $\begin{array}{l}\text { start>=0 \&\& } \\
\text { start<=s.length() }\end{array}$ & $\begin{array}{l}\text { start>=0 \&\& } \\
\text { start<=s.length() }\end{array}$ & $\begin{array}{l}\arg 0>=0 \text { \&\& } \\
\arg 0<=\text { rcv.length() }\end{array}$ \\
\hline log.substring(index) & $\begin{array}{l}\text {-1<index \&\& } \\
\text { index<log.length()+1 }\end{array}$ & $\begin{array}{l}-1<\text { index \&\& } \\
\text { index<log.length()+1 }\end{array}$ & $\begin{array}{l}-1<\arg 0 \text { \&\& } \\
\arg 0<\text { rcv.length()+1 }\end{array}$ \\
\hline $\begin{array}{l}\text { f.substring( } \\
\text { f.indexOf(“/”)) }\end{array}$ & $\begin{array}{l}\text { dir!=null \&\& } \\
\text { f.indexOf(“/”)>=0 \&\& } \\
\text { f.indexOf(“/”)<=f.length() }\end{array}$ & $\begin{array}{l}\text { true \&\& } \\
\text { f.indexOf(“/”)>=0 \&\& } \\
\text { f.indexOf(“/”)<=f.length() }\end{array}$ & $\begin{array}{l}\operatorname{true~\& \& ~} \\
\arg 0>=0 \text { \& \& } \\
\arg 0 \text { rcv.length() }\end{array}$ \\
\hline
\end{tabular}

Table 2: Example guard conditions of String. substring. API Call shows three example call sites. Guard shows the guard condition associated with each call site. Generalized shows the guard conditions after eliminating project-specific predicates. Symbolized shows the guard conditions after symbolizing variable names.

EXAMPLECHECK removes project-specific predicates (i.e., predicates that do not mention the receiver object or input arguments of the given API call) by substituting them with true. This ensures that the generalized guard condition is still implied by the original guard after removing project-specific predicates. In addition, since each code snippet may use different variable names, we normalize these names in the guard conditions. EXAMPLECHECK uses rcv and argi as the symbolic names of the receiver and the i-th input argument.

Table 2 illustrates how we canonicalize guard conditions of String. substring. This method takes an integer index as input and returns a substring that begins from the given index. The third guard condition in Column Guard contains a project-specific predicate, dir!=null. Since such predicate is not related to String. substring's arguments or receiver object, EXAMPLECHECK substitutes dir !=null with true, as shown in Column Generalized. All three examples name the receiver object differently-s, log, and f respectively. ExAMPLECHECK replaces them with a unique symbol, rcv. Similarly, EXAMPLECHECK replaces the input argument with arg0, as shown in Column Symbolized.

EXAMPLECHECK initializes each cluster with each canonicalized guard. In the following clustering process, EXAMPLECHECK checks the equivalence of every pair of clusters and merges them with if the guards are logically equivalent, until no more clusters can be merged. At the end, we count the number of guard conditions in each cluster as frequency. In a large corpus, the same logic predicate can be expressed in multiple ways. EXAMPLECHECK checks the semantic equivalence of guard conditions, instead of syntactic similarity only. EXAMPLECHECK formalizes the equivalence of two guard conditions as a satisfiability problem:

$$
p \Leftrightarrow q \text { is valid iff. } \neg((\neg p \vee q) \wedge(p \vee \neg q)) \text { is unsatisfiable. }
$$

EXAMPLECHeck uses a SMT solver, Z3 [6] to check the logical equivalence between two guards during the merging process. As Z3 only supports primitive types, ExAMPLECHECK declares variables of unsupported data types as integer variables and substitutes constants such as null with integers in Z3 queries. In addition, ExAMPLECHECK substitutes API calls in a predicate to symbolic variables based on their return types. Compared with prior work [18], ExAMPLECHECK is capable of proving the semantic equivalence of arbitrary predicates regardless of their syntactic similarity. For example, the symbolized guards of the first two examples in Table 2 are equivalent, even though they are expressed in different ways, $(-1<\arg 0$ 
\&\& $\arg \theta<r c v$. length ()$+1)$ and $(\theta<=\arg \theta \& \& \arg \theta<=r c v$. length ()$)$ respectively. Prior work [18] cannot reason about the equivalence between $-1<\arg \theta$ and $0<=\arg \theta$. However, ExAMPLECHEcK groups these logically equivalent predicates into the same cluster using the integer theorem prover in Z3.

If EXAMPLECHECK identifies a sequence pattern containing multiple guard patterns for each API call, EXAMPLECHECK enumerates different guards for each API and ranks these patterns by the number of supporting code examples in the corpora. Similar to the subsequence mining in Phase 2, ExAMPLECHECK uses a minimum support threshold $\theta$ to filter infrequent guard conditions.

We bootstrap EXAMPLECHECK with both the sequence mining threshold $\sigma$ and the guard condition mining threshold $\theta$ set to 0.5 , which means sequence and guard condition patterns are reported, only if more than half of relevant GitHub snippets include them. If EXAMPLECHeck learns no patterns with these initial thresholds, we gradually decrease both thresholds by 0.1 till finding patterns. If the mining process does not terminate after 2 hours due to too many candidate patterns, we kill the process and increase both thresholds by 0.1 accordingly. This threshold adjustment method is empirically effective to achieve a good precision (73\%).

\subsection{Manual Inspection of Mined API Usage}

EXAMPLECHECK scans over $380 \mathrm{~K}$ GitHub projects and finds an average of 55144 relevant code snippets for each API method, ranging from 211 to 450,358 snippets. This result indicates that massive corpora can provide sufficient code snippets to learn API usage patterns from. EXAMPLECHECK infers 245 API usage patterns for the 100 APIs in our study scope. This initial set of patterns may include invalid or incorrect patterns. Therefore, we manually inspect the 245 inferred patterns carefully and exclude incorrect ones based on online documentation and pattern frequencies. The overall precision is $73 \%$, resulting in 180 validated, correct patterns that we can use for the empirical study in Section 4. These 180 validated patterns cover 85 of the 100 API methods. The rest 15 API methods do not converge to any API usage patterns that can be confirmed by online documentation, since they are simple to use and do not require additional guard conditions or additional API calls. For example, System. nanoTime can be used stand-alone to obtain the current system time. Even though these 15 API methods do not have any patterns, we still include them in the scope of Stack Overflow study, since they represent a category of simple API methods that programmers are less likely to make mistakes.

During the inspection process, each pattern is annotated as either alternative or required. A code snippet should satisfy one of alternative patterns and must satisfy all required patterns. For example, EXAMPLECHEck learns firstKey()@rcv.size() $>0$ and firstKey()@!rcv.isEmpty() for SortedMap. firstKey. Both patterns ensure that a sorted map is not empty before getting the first key to avoid NoSuchElementException. They are considered alternative to each other. As an example of required patterns, programmers must handle potential IOException, when reading from a stream (e.g., Filechannel), and close it to avoid resource leaks.

Table 3 shows 25 samples of validated API patterns in 9 domains. Alternative patterns are marked with *. Column Description describes each pattern. For instance, TypedArray is allocated from a static pool to store the layout attributes, whenever a new application view is created in Android. It should be recycled immediately to avoid resource leaks and GC overhead, as mentioned in the JavaDoc. ${ }^{4}$ This pattern is supported by 2126 of 3348 related snippets in GitHub and inferred by ExAmPLECHeck (ranked \#1). The entire data set of API usage patterns for all 100 APIs and the list of SO posts with potential API usage violations are publicly available. ${ }^{5}$

\section{API MISUSE STUDY ON STACK OVERFLOW}

We use the data set of validated, desirable API usage patterns from Section 3 and study API misuse in Stack Overflow posts.

\subsection{Data Collection}

We collect all Stack Overflow posts relevant to the 100 Java APIs in our study scope from the Stack Overflow data dump. We extract code examples in the markdown <code> from SO posts with the Java tag and consider code examples in the answer posts only, since code appearing in the question posts is buggy and rarely used as examples. We gather additional information associated with each post, including view counts, vote scores (i.e., upvotes minus downvotes), and whether a post is accepted as a correct answer.

Previous studies have shown that online code snippets are often unparsable [23, 37] and contain ambiguous API elements [5] due to the incompleteness of these snippets. ExAMPLECHECK leverages a state-of-the-art partial program parsing and type resolution technique to handle these incomplete snippets, whose accuracy of API resolution is reported to be $97 \%$ [24]. Code examples that call overridden APIs or ambiguous APIs (i.e., APIs with the same name but from different Java classes) are filtered by checking the argument and receiver types respectively. In total, we find 217,818 SO posts with code examples for the 100 APIs in our study scope. Each post has 7644 view counts on average.

EXAMPLECHECK checks whether the structured call sequence of a Stack Overflow code example is subsumed by the desirable API usage in the pattern set. A structured call sequence $s$ is subsumed by a pattern $p$, only if $p$ is a subsequence of $s$ and the guard condition of each API call in $s$ implies the guard of the corresponding API call in $p$. During this subsumption checking process, the guard conditions in Stack Overflow code examples are generalized in the same manner before checking logical implication using Z3. For a SO post with multiple method-level code snippets, EXAMPLECHECK inlines invoked methods before extracting the structured call sequence in order to emulate a lightweight inter-procedural analysis.

\subsection{Manual Inspection of Stack Overflow}

To check whether Stack Overflow posts with potential API misuse reported by EXAMPLECHECK indeed suggest undesirable API usage, the first and the third authors manually check 400 random samples of SO posts with reported API usage violations. We read the text descriptions and comments of each post and check whether the surrounding narrative discusses how to prevent the violated pattern. If there are multiple code snippets in a post, we first combine them all together and check them as a single code example. We also account for aliasing during code inspection. We examine whether

\footnotetext{
${ }^{4}$ https://developer.android.com/reference/android/content/res/TypedArray.html ${ }^{5} \mathrm{http}: / /$ web.cs.ucla.edu/ tianyi.zhang/examplecheck.html
} 


\begin{tabular}{|c|c|c|c|c|}
\hline Domain & API & Pattern & Support & Description \\
\hline \multirow{2}{*}{ Collection } & ArrayList.get & loop \{; get(int)@arg0<rcv.size(); \} & 31254 & check if the index is out of bounds \\
\hline & Iterator.next & iterator()@true; loop \{; next()@rcv.hasNext();\} & 218962 & check if more elements exist to avoid NoSuchElementException \\
\hline \multirow{4}{*}{ IO } & File.createNewFile & if $\{$; createNewFile()@!rcv.exists(); $\}$ ^ & 5493 & check if the file exists before creating it \\
\hline & File.mkdir & mkdirs()@true & 26343 & call mkdirs instead, which also create non-existent parent directories \\
\hline & FileChannel.write & try \{; write(ByteBuffer)@true; close()@true; \}; catch(IOException) $\{$,$\} *$ & 1267 & close the FileChannel after writing to avoid resource leak \\
\hline & PrintWriter.write & try $\{;$ write(String)@true; close()@true; $\} ;$ catch(Exception) $\{;\}$ & 2473 & close the Printwriter after writing to avoid resource leak \\
\hline \multirow{3}{*}{ String } & StringTokenizer.nextToken & nextToken()@rcv.hasMoreTokens() * & 36179 & check if more tokens exist to avoid NoSuchElementException \\
\hline & Scanner.nextLine & loop \{; nextLine()@rcv.hasNextLine(); \} & 2510 & check if more lines exist to avoid NoSuchElementException \\
\hline & String.charAt & charAt(int)@arg0<rcv.length() & 27597 & check if the index is out of bounds \\
\hline \multirow{2}{*}{ Regex } & Matcher.find & matcher(String)@true; find()@true & 5851 & call matcher to create a Matcher instance first \\
\hline & Matcher.group & if $\{$; group(int)@rcv.find(); \} & 16447 & check if there is a match first to avoid IllegalstateException \\
\hline \multirow{3}{*}{ Database } & SQLiteDatabase.query & query(String,String[],String,String[],String,String,String)@true; close()@true & 5563 & close the cursor returned by query to avoid resource leak \\
\hline & ResultSet.getString & try $\{;$ getString(String)@rcv.next(); $\} ;$ catch(Exception) $\{;\}$ & 18933 & check if more results exist to avoid SQLException \\
\hline & Cursor.close & finally \{; close()@rcv!=null; \} & 15732 & call close in a finally block \\
\hline \multirow{4}{*}{ Android } & Activity.setContentView & onCreate(Bundle)@true; setContentView(View)@true & 56321 & always call super. oncreate first to avoid exceptions \\
\hline & TypedArray.getString & getString(int)@true; recycle()@true & 2126 & recycle the TypedArray so it can be reused by a later call \\
\hline & SharedPreferences.Editor.edit & edit()@true; commit()@true & 9650 & commit the changes to SharedPreferences \\
\hline & ApplicationInfo.loadIcon & getPackageManager()@true; loadIcon(PackageManager)@true & 400 & get a Packagemanager as the argument of load \\
\hline \multirow[t]{2}{*}{ Crypto } & Mac.doFinal & $\begin{array}{l}\text { try \{; getInstance(String)@true; getBytes()@true; doFinal(byte[])@true; } \\
\} ; \text { catch(Exception) }\{;\}\end{array}$ & 474 & get a Mac instance first and convert the input to bytes \\
\hline & MessageDigest.digest & getInstance(String)@true; digest()@true & 7048 & get a MessageDigest instance first \\
\hline \multirow{3}{*}{ Network } & Jsoup.connect & try $\{;$ connect(String)@true; get()@true; $\} ;$ catch(IOException); \{\} * & 376 & call get to fetch the web content \\
\hline & URL.openConnection & try $\{;$ new URL(String)@true; openConnection()@true; $\} ;$ catch(Exception) $\{;\}$ & 19056 & create a URL object first and handle potential exceptions \\
\hline & HttpClient.execute & new HttpGet(String)@true; execute(HttpGet)@true & 2536 & create a HttpGet object as the argument of execute \\
\hline \multirow{2}{*}{ Swing } & SwingUtilities.invokeLater & new Runnable()@true; invokeLater(Runnable)@true & 20406 & create a Runnable object as the argument of invokeLater \\
\hline & JFrame.setPreferredSize & setPreferredSize(Dimension)@true, pack()@true & 394 & call pack to update the JFrame with the preferred size \\
\hline
\end{tabular}

Table 3: 25 samples of manually validated API usage patterns after GitHub code mining.

the reported API usage violation could produce any potential behavior anomaly, such as program crashes and resource leaks on a contrived input data or program state and whether such anomaly could have been eliminated by following the desirable pattern. For short posts, this inspection takes about 5 minutes each. For longer posts with a big chunk of code or multiple code fragments, it takes around 15 to 20 minutes. To reduce subjectivity, the two authors inspect these posts independently. The initial inter-rater agreement is 0.84 , measured by Cohen's kappa coefficient [32]. The two authors resolve disagreements on all but two posts, and the kappa coefficient after the discussion is 0.99 . The two authors disagree how helpful reported violations are in two posts, where API usage violations in these posts are either clarified in surrounding natural language explanations or mentioned in post comments.

True Positive. 289 out of 400 inspected Stack Overflow posts $(72 \%)$ contain real API misuse, confirmed by both authors. For instance, the following example demonstrates how to retrieve records from SQLiteDatabase using Cursor but forgets to close the database connection at the end. ${ }^{6}$ Programmers should always close the connection to release all its resources. Otherwise, it may quickly run out of memory, when retrieving a large volume of data from the database frequently.

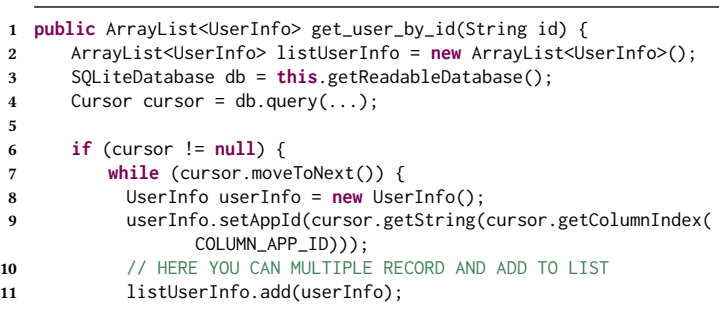

$\overline{{ }^{6} \text { https://stackoverflow.com/questions/31531250 }}$

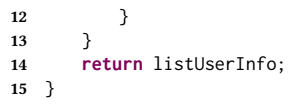

In many cases, a code example may function well with some crafted input data, even though it does not follow desirable API usage. For example, programmers should check whether the return value of String. index of is negative to avoid IndexOutOfBoundsException. The example below does not follow this practice, but still works well with a hard-coded constant, text. ${ }^{7}$ One can argue that the input data is hard-coded for illustration purposes only, as the role of Stack Overflow post is to provide a starting point rather than teaching complete details of correct API usage. However, if a programmer reuses this code example and replaces the hard-coded text with a function call reading from a $\mathrm{html}$ file, the reused code may crash if the html document does not have an expected element. Therefore, it is still beneficial to inform the users about desirable usage and potential pitfalls, especially for a novice programmer who may not be familiar with the given API.

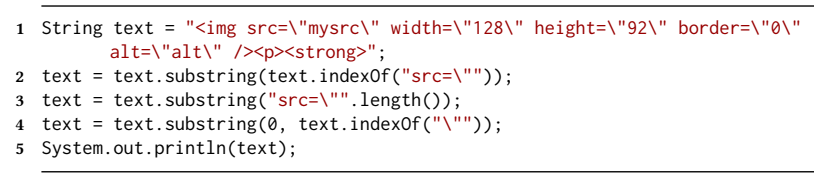

FALSe Positive. ExAmpleCheck mistakenly detects API misuse in 64 posts. The majority reason is that EXAMPLECHECK checks for API misuse via a sequence comparison without deep knowledge of its specification, which is not sufficient in 56 posts. For instance, the following SO post calls substring (line 5 ) without explicitly checking whether the start index (index+1) is not a negative number and the end index (strvalue. length()) is not greater than the length

\footnotetext{
${ }^{7}$ https://stackoverflow.com/questions/12742734
} 
of the string. ${ }^{8}$ While EXAMPLECHECK warns potential API misuse, according to JDK specifications, indexof never returns a negative integer $\leq-2$. Thus, the following code is still safe, because index +1 is guaranteed to be non-negative. Similarly, strvalue. length() returns the string's length, which cannot be out of bounds. Such cases require having detailed specifications, such as the return value of index $O f()$ is always $\geq 1$.

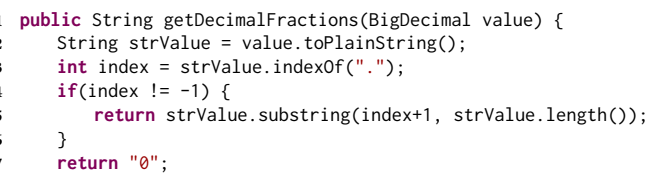

Second, 36 false positives are correct but infrequent alternatives. EXAMPLECHEсK does not learn these alternative usage patterns, because they do not commonly appear in GitHub. For example, programmers should first call new SimpleDateFormat to instantiate SimpleDateFormat with a valid date pattern and then call format, which is supported by 18,977 related GitHub snippets. An alternative way is to instantiate SimpleDateFormat by calling getInstance, as shown in the following SO post. ${ }^{9}$ This alternative usage is supported by 360 GitHub snippets and therefore not inferred by ExAMPLECHECK due to its low frequency.

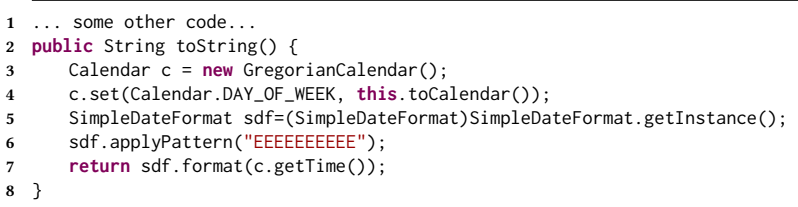

In some SO posts, users explicitly state in surrounding natural language text that the given code example must be improved during integration or adaptation. The following example shows how to load a Class instance by name and then cast the class. ${ }^{10}$ The author of this post comments that "be aware that this might throw several Exceptions, e.g. if the class defined by the string does not exist or if AnotherClass. classMethod() doesn't return an instance of the class you want to cast to." EXAMPLECHECK still flags the post because of a missing exception handling, since the desirable API usage is not reflected in the embedded code. However, it is certainly possible that $\mathrm{SO}$ users will read both the code and surrounding text carefully and investigate how to handle edge cases narrated in the text.

1 Class $<$ ? $>$ myclass = Class. forName ("myClass_t" $)$;

2 myClass_t myVar $=($ myClass_t $)$ myclass. cast $($ AnotherClass. classMethod ()$)$;

Sometimes, Stack Overflow users split a single code example into multiple fragments and provide step-by-step explanation, which is considered as a better way of answering questions in Stack Overflow [17]. EXAMPLECHECK may report API misuse if two related API calls are split in different code fragments. ${ }^{11}$ This can be addressed by stitching these snippets together during analysis.

\footnotetext{
${ }^{8}$ http://stackoverflow.com/questions/7473462

${ }^{9}$ https://stackoverflow.com/questions/2243850

${ }^{10} \mathrm{https} / / /$ stackoverflow.com/questions/4650708

${ }^{11} \mathrm{https} / / /$ stackoverflow.com/questions/11552754
}

\subsection{Is API Misuse Prevalent on Stack Overflow?}

EXAMPLECHECK detects potential API misuse in 66,897 (31\%) out of 217,818 Stack Overflow posts in our study. We manually label each API pattern with its corresponding domain as well as the consequence of each possible violation. Then we write scripts to categorize reported violations based on their domains and based on their consequences. Figure 4 shows the prevalence of API misuse from different domains. Database, IO, and network APIs are often misused, since they often require to handle potential runtime exceptions and close underlying streams to release resources properly at the end. Similarly, many cryptography related posts are flagged as unreliable, due to unhandled exceptions. Stack Overflow posts on string and text manipulation often forget to check the validity of input data (e.g., whether the input string is empty) or return values (e.g., whether the returned character index is -1).

Among posts with potential API misuse reported by ExAMPLECHECK, 76\% could potentially lead to program crashes, e.g., unhandled runtime exceptions. $18 \%$ could lead to incomplete action, e.g., not completing a transaction after modifying resources in Android, or not calling setvisible after modifying the look and feel of a swing GUI widget. $2 \%$ could lead to resource leaks in operating systems, e.g., not closing a stream. We fully acknowledge that not all detected violations could lead to bugs when ported to a target application. To accurately assess the runtime impact of SO code examples, one must systematically integrate these examples to real-world target applications and run regression tests.

Many SO examples aim to answer a particular programming question. Therefore, authors of these examples may assume SO users who posted these questions already know about the used APIs and may not include complete details of desirable API usage. However, given that each post has 7,644 view counts on average, some users may not have similar background knowledge. Especially for novice programmers, it may be useful to show extra tips about desirable API usage evidenced by a large number of GitHub code snippets. We also find that SO posts with API misuse are more frequently viewed than those posts without API misuse, 8365 vs. 7276 on average. Therefore, there is an opportunity to help users consider better or alternative API usage mined from massive corpora, when they stumble upon SO posts with potential API misuse.

\subsection{Are highly voted posts more reliable?}

Stack Overflow allows users to upvote and downvote a post to indicate the applicability and usefulness of the post. Therefore, votes are often considered the main quality metric of Stack Overflow examples [17]. However, we find that highly voted posts are not necessarily more reliable in terms of correct API usage. Figure 5 shows the percentage of SO posts with different vote scores that are detected with at least one API usage violation. We perform a linear regression on the vote score and the percentage of unreliable examples, as shown by the red line in Figure 5 . We do not observe a strong positive or negative correlation between the vote of a post and its reliability in terms of API misuse. A previous study shows that concise code examples and detailed step-by-step explanations are two key factors of highly voted Stack Overflow posts [17]. Our manual inspection confirms that many unreliable examples are simplified to operate on crafted input data for illustration purposes only 


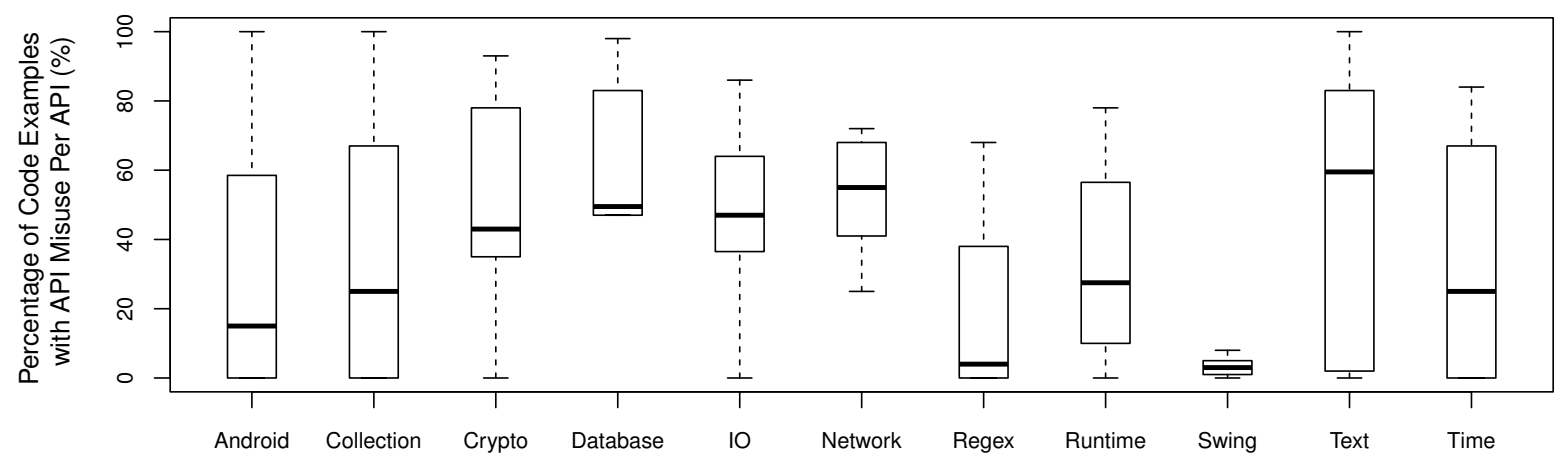

Figure 4: API Misuse Comparison between Different Domains

(Section 4.2). Such curated examples are not sufficient for various input data and usage scenarios in real software systems, especially for handling corner cases. Therefore, votes alone should not be used as a single indicator of the quality of online code examples. To improve the quality of curated examples, Stack Overflow needs another mechanism that helps developers understand the limitation of existing examples and decide how to integrate the given example to production code (Section 5).

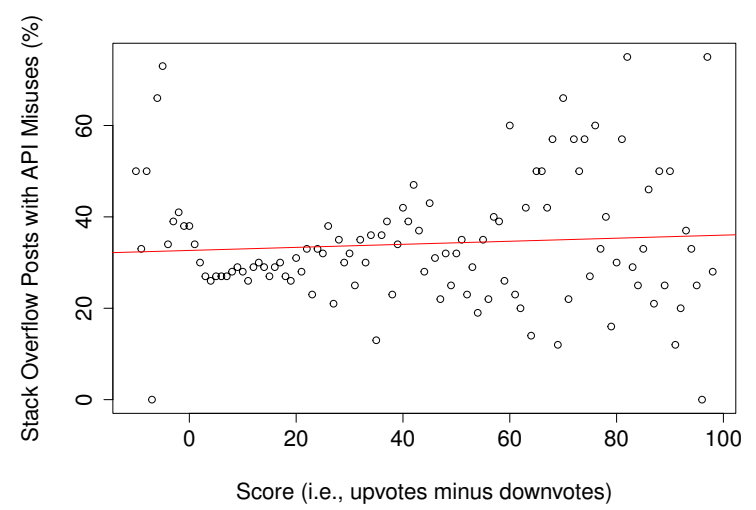

Figure 5: API Misuse Comparison between Code Examples with Different Vote Scores on Stack Overflow

\subsection{What are the characteristics of API misuse?}

We classify the detected API usage violations into three categories based on the required edits to correct the violations.

Missing Control Constructs. Many APIs should be used in a specific control-flow context to avoid unexpected behavior. This type of API usage violations can be further split based on the type of missing control constructs.

Missing exception handling. If an API may throw an exception, the thrown exception should either be caught and handled a try-catch block or be declared in the method header. In total, we find 17,432 code examples that do not handle exceptions properly. For example, Integer. parseInt may throw NumberFormatException if the string does not contain a parsable integer. The following example will crash, if a user enters an invalid integer. ${ }^{12}$ A good practice is to surround parseInt with a try-catch block to handle the potential exception. Unlike checked exceptions such as IOException, runtime exceptions such as NumberFormatException will not be checked at compile time. In such cases, it would be helpful to inform users about which runtime exceptions must be handled based on common exception handling usage in GitHub.

1 Scanner scanner = new Scanner (System.in);

2 System. out.print("Enter Number of Students: $\backslash t ")$;

3 int numStudents $=$ Integer . parseInt (scanner $\cdot$ nextLine ()$)$;

Missing if checks. Some APIs may return erroneous values such as null pointers, which must be checked properly to avoid crashing the succeeding execution. For example, TypedArray.getString may return null, if the given attribute is not defined in the style resource of an Android application. Therefore, the return value, customfont must be checked before passing it as an argument of setCustomfont (line 6) to avoid NullPointerException, which is violated by the following Stack Overflow example. ${ }^{13}$

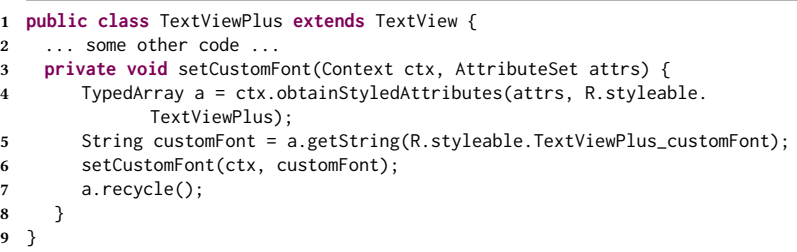

Missing finally. Clean-up APIs such as close should be invoked in a finally block in case an exception occurs before invoking those APIs. 83\% of Stack Overflow examples that call Cursor. close does not call it in a finally block, shown in the following. ${ }^{14}$ Cursor. close may be skipped, if getString (line 5) throws an exception.

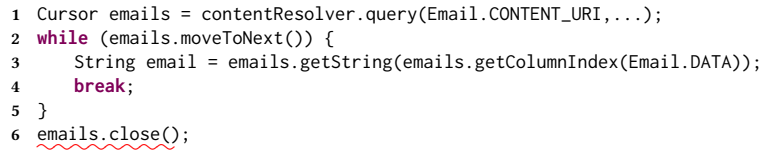

Missing or Incorrect Order of API calls. In certain cases, multiple APIs should be called together in a specific order to achieve

\footnotetext{
${ }^{12} \mathrm{https} / / /$ stackoverflow.com/questions/3137481

${ }^{13}$ https://stackoverflow.com/questions/7197867

${ }^{14}$ https://stackoverflow.com/questions/31427468
} 


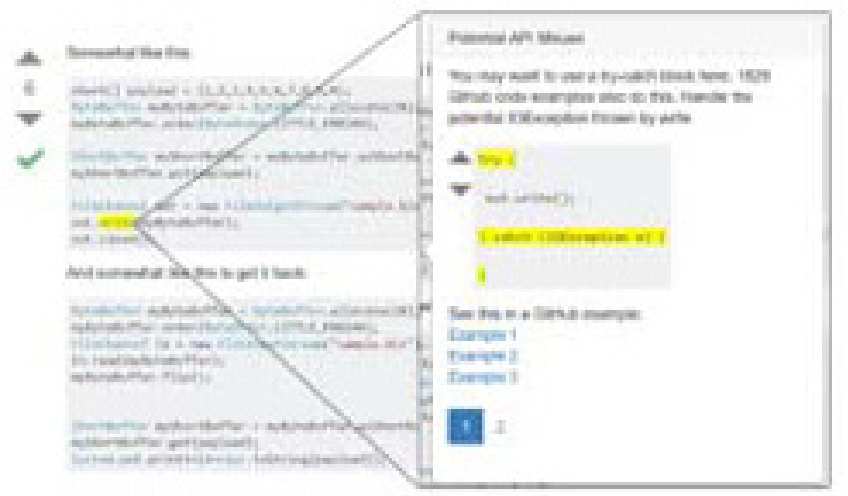

Figure 6: Chrome extension for augmenting Stack Overflow posts with mined API usage

desired functionality. Missing or ineorrect order of such API calls can lead to unexpected behavior. For example, developers must call flip, rewind, or position to reset the internal cursor of Bytebuffer back to the previous position to read the buffered data properly The following SO example could throw Buffer Underflowe xoept icn. if the internal cursor already reached the upper bound of the buffer after the put operation at line $2^{15}$ Without resetting the internal cursor, the next got Int operation at line 3 would start reading from the upper bound, which is prohibited. We find 7,956 posts that either misses a critical API call or calls APIs in an incorrect order.

1. Eycubuffer ba = Antwouffer -allacate(4);

I ib. outinestrat):

int $1 \cdot$ to patrato

Incorrect Guard Conditions. Many AFls should be invoked under the cortect guard condition to avoid runtime exceptions. For instance, programmers shuuld check whether a sorted map is empty with a guard like asp.size () 38 or I nap. isEapty () before calling firstKey (APIv) on the map. However, the following calls firstKey on an empty matp without a guand, leading to NoFuch El lenentE acept ien. ${ }^{16}$ Surprisingly, this example is accepled as the correct answer and also upvoted by six other developers on Stack Overflow, We find 13,791 posts with incorrect gaard conditions.

t. Treetap nas * ane Tremesac

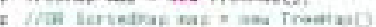

ing furstess

\section{DISCUSSION}

Augmentation of Stack Overflow. The study results from Sec. tion 4 indicale that even highly voted and frequently viewed SO posts do not necessarily follow desirable API usage. There is an opportunity to help developers consider hetter ar alternative API usage that is mined from massive corpora and is supported by thoosands of GitHub snippets. Certainly, the goal of Stack Overflow is to provide 'quick snippets' and not to share complete details of API usage or present compilable, runnable code. Rather, Stack Overflow often serves the purpose of providing a starting point

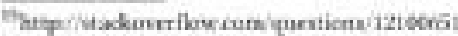

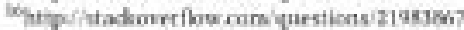

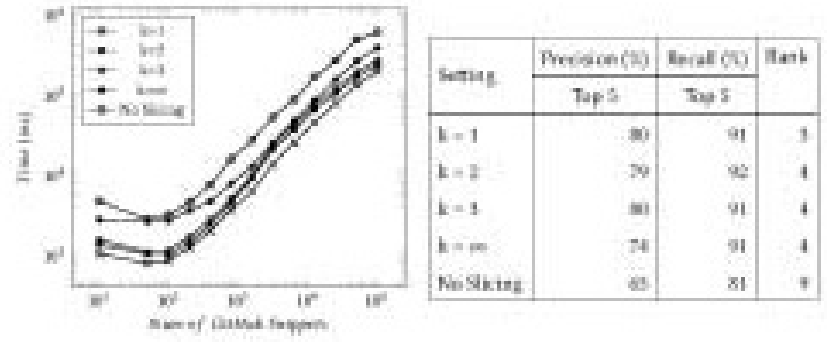

Figure 7: Mining time and accuracy with varying $k$ bounds.

and helping the user to grasp the gist of how the API works by omitting associated details sach as which guand conditions to check and which runtime exceptions to handle. Nevertheless, it would be useful for a user to see related AII usage along with concrete examples substantiating the desirable API usage, when the user is browsing the given SO post. Such information may reduce the effort of integrating, adapting, and testing the given code example.

Figure 6 sketches a Chrome extension that we design to augment at given $S O$ post with mined APl usage patterns, If there exists better or alternative API usage such as enclosing Filechannel write withis a try-catch block, the browser exteasion bighlights the relevant A.P call on the original post and provides a howering menu with the description "You may want to use ... 5839 Gathub code snippets also do this aloxg with concrete examples. While our proposed browser extension follows a similar style to Codota ${ }^{13}$ Codota does not group related examples based on common API usage, does not quantify how many GitHub code snippets support the comman usage, and does rot detect API misuse by contrasting the SO post against the usage. We leverage the data set of GitHub snippets mined by Exastrix aresc and design novel interactive visualization for exploring massive code examples simultuneously [12].

API Usage Mining Running Time and Accuracy. We brielly describe the ruming time and nccuracy of APl usage mining employed in Maple. Figure $7\{a\}$ shows the performance of ExasurizCarck with different $k$ hounds. On average, the mining time is within 10 minutes for each AIL. We run each experiment five times and compate the average execution time. Setting $k$ to $\infty 0$ retains all dependent API calls in a sticed call sequence, while setting $k$ to 1 retains only immediately dependent calls. Setting $k$ to 1 can achieve $3.3 \mathrm{X}$ speed up compared with selting $\mathrm{k}$ to ox, since it creales shorter call sequences by removing transitively dependent AIl calls. EXAMPLECHECK rans op to $4.6 \mathrm{X}$ slower without program slicing.

Figure $7\langle b\rangle$ shows the pattern mining accuracy using different $k$ bounds. The evaluation is done for the 30 APls from MUBENCH asing its grooud truth [2]. Exastple CerEck has $80 \%$ precision and 91\% recall, when considering top 5 patterns for each API method. Even though limiting dependency analysis wath lower bounds may lead to incomplete sequences with fewer API calls. varying $k$ does not affect accuracy much. However, compared with unbounded anatysis, filtering out transitively depeodent APl ealls can improwe precision aod recall sliphtly. This is becuuse long API call sequences may introduce additional patterns of no interest.

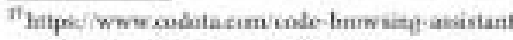


Threats to Validity. Our study is limited to 100 Java APIs that frequently appear in Stack Overflow and thus may not generalize to other Java APIs or different languages. Our scope is limited to code snippets found on Stack Overflow. Other types of online resources such as programming blogs and other Q\&A forums may have better curated examples. According to the manual inspection of 400 sampled SO posts with detected API usage violations, ExAMPLECHECK detects API misuse with 72\% precision (Section 4.2). While the precision is rather low, EXAMPLECHECK could be still useful in the case of false positives, since the goal of EXAMPLECHECK is not to discard SO posts with potential API violations, but rather to suggest desirable or alternative API usage details to the users.

\section{RELATED WORK}

Quality Assessment of Online Code Examples. Prior work has investigated the quality of online code examples from different perspectives. The majority of code examples on Stack Overflow are free-standing program statements that cannot be accepted by compilers [23, 37]. Due to the incompleteness of code snippets, $89 \%$ of API names in code snippets from online forums are ambiguous and cannot be easily resolved [5]. Subramanian et al. design a partial program parsing and type resolution technique for Stack Overflow code snippets, which EXAMPLECHECK uses to resolve API names [24]. Zhou et al. find that 86 of 200 accepted posts on Stack Overflow use deprecated APIs but only 3 of them are reported by other programmers [39]. Fischer et al. investigate security-related code on Stack Overflow and find that $29 \%$ is insecure [9]. They further apply clone detection to check whether insecure code is reused from Stack Overflow to Android applications on Google Play and find that insecure code may have been copied to over 1 million Android apps. An et al. investigate copyright issues between Stack Overflow and GitHub [4] and find a large number of potential license violations. Treude and Robillard conduct a survey to investigate comprehension difficulty of code examples in Stack Overflow [29]. The responses from GitHub users indicate that less than half of the SO examples are self-explanatory.

While our study also indicates the limitation of code example quality in Stack Overflow, our study focuses on API usage violations that may lead to unexpected behavior such as program crashes and resource leaks by contrasting SO code examples against desirable API usage mined from massive corpora. Our results strongly motivate the need of systematically augmenting Stack Overflow and helping the user to implicitly assess the given SO example with quantitative evidence about how many GitHub snippets follow (or do not follow) related API usage patterns.

API Usage Mining. There is a large body of literature in mining implicit programming rules, API usage, and temporal properties of API calls. Since API usage mining is only a part of our data set construction process, we are not arguing the novelty of API mining employed in EXAMPLECHECK. Nevertheless, we briefly describe how API usage mining in EXAMPLECHECK is related to prior work.

Gruska et al. extract call sequences from programs and perform formal concept analysis [11] to infer pairwise temporal properties of API calls [14]. Many other specification mining techniques are dedicated to inferring temporal properties of API calls $[3,8,10$, 19, 20, 35]. UP-Miner mines frequent sequence patterns but does not retain control constructs and guard conditions in API usage patterns [33]. Several techniques [15, 16, 27] model programs as item sets and infer pairwise programming rules using frequent itemset mining [13], which does not consider temporal ordering or guard conditions of API calls.

EXAMPLECHECK mines from massive corpora of GitHub projects, several orders of magnitude larger than prior work [14, 20,33, 35]. EXAMPLECHECK mines not only API call ordering but also guard conditions using predicate mining. To our best knowledge, Ramanathan et al. [21] and Nguyen et al. [18] are the only two predicate mining techniques. Ramanathan et al. apply inter-procedure data-flow analysis to collect all predicates related to a call site and then use frequent itemset mining to find common predicates. Unlike ExAMPLECHECK, Ramanathan et al. only mine a single project and cannot handle semantically equivalent predicates in different forms. Nguyen et al. improve upon Ramanathan et al. by normalizing predicates using several rewriting heuristics. Unlike these techniques, EXAMPLECHECK formalizes the predicate equivalence problem as a satisfiability problem and leverages a SMT solver to group logically equivalent predicates during guard mining.

\section{CONCLUSION}

Programmers often resort to code examples on online Q\&A forums such as Stack Overflow to learn about how to use APIs correctly during software development. However, the reliability of code examples in Stack Overflow posts is under-investigated. To demonstrate the prevalence and severity of API misuse in online code examples, we mine frequent API usage patterns from 380,125 GitHub repositories, carefully check the resulting 245 mined patterns manually, and contrast 217,818 Stack Overflow posts with 180 validated patterns. Our study provides empirical evidence that almost one third of Stack Overflow posts may contain potential API usage violations that could produce symptoms such as program crashes and resource leaks. Even highly voted posts are not necessarily more reliable than other posts in terms of API usage correctness.

Certainly, the purpose of Stack Overflow is to provide a starting point for investigation and its code examples do not necessarily include all details of how to reuse the given code. However, for novice developers, it may be useful to show extra tips about desirable API usage evidenced by a large number of GitHub snippets. Our work provides a foundation for enriching and enhancing code snippets in a collaborative Q\&A forum by contrasting them against frequent usage patterns learned from massive code corpora. Such approach could help the user to implicitly assess the given code example and reduce the effort of integrating, adapting, and testing the curated example in a target application. As a future work, we plan to validate EXAMPLECHECK with developers and solicit their feedback on its Chrome extension.

\section{ACKNOWLEDGMENTS}

We would like to thank anonymous reviewers for the helpful feedback. This work is supported by AFRL grant FA8750-15-2-0075, and NSF grants CCF-1527923, CCF-1460325, CCF-1423370, CNS1513263, and CCF-1518897. Anastasia Reinhardt's internship at UCLA is supported by CRA-W Distributed Research Experiences for Undergraduates (DREU) program. 


\section{REFERENCES}

[1] Frances E Allen. 1970. Control flow analysis. In ACM Sigplan Notices, Vol. 5. ACM, 1-19.

[2] Sven Amani, Sarah Nadi, Hoan A Nguyen, Tien N Nguyen, and Mira Mezini. 2016. MUBench: a benchmark for API-misuse detectors. In Proceedings of the 13th International Conference on Mining Software Repositories. ACM, 464-467.

[3] Glenn Ammons, Rastislav Bodik, and James R. Larus. 2002. Mining specifications. In POPL '02: Proceedings of the 29th ACM SIGPLAN-SIGACT Symposium on Principles of Programming Languages. ACM Press, New York, NY, USA, 4-16. DOI : http://dx.doi.org/10.1145/503272.503275

[4] Le An, Ons Mlouki, Foutse Khomh, and Giuliano Antoniol. 2017. Stack overflow: a code laundering platform?. In Software Analysis, Evolution and Reengineering (SANER), 2017 IEEE 24th International Conference on. IEEE, 283-293.

[5] Barthélémy Dagenais and Martin P Robillard. 2012. Recovering traceability links between an API and its learning resources. In 2012 34th International Conference on Software Engineering (ICSE). IEEE, 47-57.

[6] Leonardo De Moura and Nikolaj Bjørner. 2008. Z3: An efficient SMT solver. In International conference on Tools and Algorithms for the Construction and Analysis of Systems. Springer, 337-340.

[7] Robert Dyer, Hoan Anh Nguyen, Hridesh Rajan, and Tien N Nguyen. 2013. Boa: A language and infrastructure for analyzing ultra-large-scale software repositories. In Proceedings of the 2013 International Conference on Software Engineering. IEEE Press, 422-431.

[8] Michael D Ernst, Jake Cockrell, William G Griswold, and David Notkin. 2001. Dynamically discovering likely program invariants to support program evolution IEEE Transactions on Software Engineering 27, 2 (2001), 99-123.

[9] Felix Fischer, Konstantin Böttinger, Huang Xiao, Christian Stransky, Yasemin Acar, Michael Backes, and Sascha Fahl. 2017. Stack Overflow Considered Harmful? The Impact of Copy\&Paste on Android Application Security. In Security and Privacy (SP), 2017 IEEE Symposium on. IEEE, 121-136.

[10] Mark Gabel and Zhendong Su. 2010. Online inference and enforcement of temporal properties. In Proceedings of the 32nd ACM/IEEE International Conference on Software Engineering-Volume 1. ACM, 15-24.

[11] Bernhard Ganter and Rudolf Wille. 2012. Formal concept analysis: mathematical foundations. Springer Science \& Business Media.

[12] Elena Glassman*, Tianyi Zhang*, Björn Hartmann, and Miryung Kim. 2018. Visualizing API Usage Examples at Scale. In Proceedings of the SIGCHI Conference on Human Factors in Computing Systems. ACM. *The two lead authors contributed equally to the work as part of an equal collaboration between both institutions.

[13] Gösta Grahne and Jianfei Zhu. 2003. Efficiently using prefix-trees in mining frequent itemsets.. In FIMI, Vol. 90

[14] Natalie Gruska, Andrzej Wasylkowski, and Andreas Zeller. 2010. Learning from 6,000 projects: lightweight cross-project anomaly detection. In Proceedings of the 19th international symposium on Software testing and analysis. ACM, 119-130.

[15] Zhenmin Li and Yuanyuan Zhou. 2005. PR-Miner: automatically extracting implicit programming rules and detecting violations in large software code. In ACM SIGSOFT Software Engineering Notes, Vol. 30. ACM, 306-315.

[16] Martin Monperrus, Marcel Bruch, and Mira Mezini. 2010. Detecting missing method calls in object-oriented software. In European Conference on ObjectOriented Programming. Springer, 2-25.

[17] Seyed Mehdi Nasehi, Jonathan Sillito, Frank Maurer, and Chris Burns. 2012. What makes a good code example?: A study of programming Q\&A in StackOverflow. In Software Maintenance (ICSM), 2012 28th IEEE International Conference on. IEEE, 25-34.

[18] Hoan Anh Nguyen, Robert Dyer, Tien N Nguyen, and Hridesh Rajan. 2014. Mining preconditions of APIs in large-scale code corpus. In Proceedings of the 22nd ACM SIGSOFT International Symposium on Foundations of Software Engineering. ACM, 166-177.

[19] Michael Pradel and Thomas R Gross. 2009. Automatic generation of object usage specifications from large method traces. In Proceedings of the 2009 IEEE/ACM International Conference on Automated Software Engineering. IEEE Computer Society, 371-382.

[20] Michael Pradel, Ciera Jaspan, Jonathan Aldrich, and Thomas R Gross. 2012. Statically checking API protocol conformance with mined multi-object specifications In Proceedings of the 34th International Conference on Software Engineering. IEEE Press, 925-935.

[21] Murali Krishna Ramanathan, Ananth Grama, and Suresh Jagannathan. 2007. Static specification inference using predicate mining. In ACM SIGPLAN Notices, Vol. 42. ACM, 123-134.

[22] Caitlin Sadowski, Kathryn T Stolee, and Sebastian Elbaum. 2015. How developers search for code: a case study. In Proceedings of the 2015 10th foint Meeting on Foundations of Software Engineering. ACM, 191-201.

[23] Siddharth Subramanian and Reid Holmes. 2013. Making sense of online code snippets. In Proceedings of the 10th Working Conference on Mining Software Repositories. IEEE Press, 85-88.

[24] Siddharth Subramanian, Laura Inozemtseva, and Reid Holmes. 2014. Live API documentation. In Proceedings of the 36th International Conference on Software
Engineering. ACM, 643-652.

[25] Suresh Thummalapenta and Tao Xie. 2007. Parseweb: a programmer assistant for reusing open source code on the web. In Proceedings of the twenty-second IEEE/ACM international conference on Automated software engineering. ACM, 204-213.

[26] Suresh Thummalapenta and Tao Xie. 2009. Mining exception-handling rules as sequence association rules. In Proceedings of the 31st International Conference on Software Engineering. IEEE Computer Society, 496-506.

[27] Suresh Thummalapenta and Tao Xie. 2011. Alattin: mining alternative patterns for defect detection. Automated Software Engineering 18, 3 (2011), 293.

[28] Emina Torlak and Satish Chandra. 2010. Effective Interprocedural Resource Leak Detection. In Proceedings of the 32Nd ACM/IEEE International Conference on Software Engineering - Volume 1 (ICSE '10). ACM, New York, NY, USA, 535-544. DOI : http://dx.doi.org/10.1145/1806799.1806876

[29] Christoph Treude and Martin P Robillard. 2017. Understanding Stack Overflow Code Fragments. In Proceedings of the 33rd International Conference on Software Maintenance and Evolution. IEEE.

[30] Medha Umarji, Susan Elliott Sim, and Crista Lopes. 2008. Archetypal internetscale source code searching. In IFIP International Conference on Open Source Systems. Springer, 257-263.

[31] Ganesha Upadhyaya and Hridesh Rajan. 2018. Collective Program Analysis. In Proceedings of the 40th International Conference on Software Engineering. ACM.

[32] Anthony J Viera, Joanne M Garrett, and others. 2005. Understanding interobserver agreement: the kappa statistic. Fam Med 37, 5 (2005), 360-363.

[33] Jue Wang, Yingnong Dang, Hongyu Zhang, Kai Chen, Tao Xie, and Dongmei Zhang. 2013. Mining succinct and high-coverage API usage patterns from source code. In Proceedings of the 10th Working Conference on Mining Software Repositories. IEEE Press, 319-328.

[34] Jianyong Wang, Jiawei Han, and Chun Li. 2007. Frequent closed sequence mining without candidate maintenance. IEEE Transactions on Knowledge and Data Engineering 19, 8 (2007).

[35] Andrzej Wasylkowski, Andreas Zeller, and Christian Lindig. 2007. Detecting object usage anomalies. In Proceedings of the the 6th joint meeting of the European software engineering conference and the ACM SIGSOFT symposium on The foundations of software engineering. ACM, 35-44.

[36] Mark Weiser. 1981. Program slicing. In Proceedings of the 5th international conference on Software engineering. IEEE Press, 439-449.

[37] Di Yang, Aftab Hussain, and Cristina Videira Lopes. 2016. From query to usable code: an analysis of stack overflow code snippets. In Proceedings of the 13th International Workshop on Mining Software Repositories. ACM, 391-402.

[38] Hao Zhong, Tao Xie, Lu Zhang, Jian Pei, and Hong Mei. 2009. MAPO: Mining and recommending API usage patterns. In European Conference on Object-Oriented Programming. Springer, 318-343.

[39] Jing Zhou and Robert J Walker. 2016. API deprecation: a retrospective analysis and detection method for code examples on the web. In Proceedings of the 2016 24th ACM SIGSOFT International Symposium on Foundations of Software Engineering. ACM, 266-277. 